\title{
Synbiotics in oral immunity: a review
}

\author{
Kanishk Mehta ${ }^{1 *}$, Heena Mehta ${ }^{2}$ \\ ${ }^{1}$ Department of Ear Nose Throat and Head Neck Surgery, American International Institute of Medical Science, \\ Udaipur, Rajasthan, India \\ ${ }^{2}$ Department of Oral Medicine and Radiology, Darshan Dental College and Hospital, Udaipur, Rajasthan, India
}

Received: 26 February 2021

Revised: 14 April 2021

Accepted: 19 April 2021

\author{
*Correspondence: \\ Dr. Kanishk Mehta, \\ E-mail: drkanishkm@gmail.com
}

Copyright: () the author(s), publisher and licensee Medip Academy. This is an open-access article distributed under the terms of the Creative Commons Attribution Non-Commercial License, which permits unrestricted non-commercial use, distribution, and reproduction in any medium, provided the original work is properly cited.

\begin{abstract}
The oral cavity harbors numerous and varied microbial flora. The existence of probiotics in the indigenous oral micro flora of humans warrants exploration because these bacteria offer the advantage of being perfectly adapted to the oral ecosystem. The concept of probiotics probably dates back to 1908 when the long life of Bulgarian peasants was the result of their lifelong consumption of fermented milk products. The pursuit of original observation of the positive role played by some selected bacteria, as investigated by Elie Metchnikoff, the Ukrainian-born Nobel Prize winner working at the Pasteur Institute, at the beginning of the last century much still remains to be done to standardize the meaning of the term synbiotic and which strains actually fulfil the criteria of true synbiotic microorganisms. Since, there are established reports in its applicability, this opens up a new era of interest.
\end{abstract}

Keywords: Synbiotics, Probiotics, Prebiotics

\section{INTRODUCTION}

The oral cavity harbours numerous and varied microbial flora. Remarkably, these potentially overwhelming populations coexist within the host, with harmful effects occurring only if the immune status is altered. It is now generally accepted that this endogenous microflora possesses immuno-modulating capacities. ${ }^{1}$ This concept of beneficial bacteria has led to the advent of probiotics. The term probiotic was derived from the Greek, meaning 'for life'. Probiotics, the antonym of the term antibiotics, was introduced in 1965 by Lilly and Stillwell as, 'Substances produced by microorganisms which promote the growth of other micro-organisms'. A prebiotic agent is a nondigestible food ingredient that confers benefits on the host by selectively stimulating one bacterium or a group of bacteria in the colon with probiotic properties. Both probiotics and prebiotics are collectively known as 'synbiotics'.
Several factors are now leading many physicians to examine probiotics and other alternatives to pharmaceutical remedies. These include the surging levels of multidrug resistance among pathogenic organisms, particularly in hospitals, the increasing demands of consumers for natural substitutes for drugs, and the emergence of scientific and clinical evidence showing the efficacy and effectiveness of some probiotic strains. Synbiotics represents an expanding research area.

\section{METHODS}

Various articles were selected on the topic of probiotics, prebiotics and synbiotics online and the articles from the last 2 decades (from 2001-2020) were reviewed to keep the most updated and recent developments in the field relevant. Considering the current situation of global pandemic, the need for developing and ideal synbiotic for boosting the immunity, the entire topic was divided into following headings: (a) normal flora- the basic understanding of what a human flora consists of, its 
beneficial and harmful effects; (b) ideal properties- what according to various researchers are the ideal properties that a synbiotic compound must possess; (c) oral benefitsthe list of benefits that we desire from the ideal compound.

\section{DISCUSSION}

\section{Normal flora}

The normal flora of human are exceedingly complex and consist of more than 200 species of bacteria. The makeup of the normal flora may be influenced by various factors, including genetics, age, sex, stress, nutrition and diet of the individual. Three developmental changes in humans: weaning, the eruption of the teeth, and the onset and cessation of ovarian functions, invariably affect the composition of the normal flora in the intestinal tract, the oral cavity, and the vagina, respectively. Some of these organisms are known to perform tasks that are useful for the human host, while the majority have no known beneficial or harmful effect.

\section{Oral microflora}

The presence of nutrients, epithelial debris, and secretions makes the mouth a favourable habitat for a great variety of bacteria. Oral bacteria include streptococci, lactobacilli, staphylococci and corynebacteria, with a great number of anaerobes, especially bacteroides. The mouth presents a succession of different ecological situations with age and this corresponds with changes in the composition of the normal flora. Streptococcus salivarius is dominant and may make up $98 \%$ of the total oral flora until the appearance of the teeth. The eruption of the teeth during the first year leads to colonization by S. mutans and S. sanguis. These bacteria require a non-desquamating (nonepithelial) surface in order to colonize. The creation of the gingival crevice area increases the habitat for the variety of anaerobic species. $^{2}$

\section{Beneficial effects of the normal flora}

Synthesis and excretion of vitamins in excess of their own needs, which can be absorbed as nutrients by their host; prevention of colonization by pathogens by competing for attachment sites or for essential nutrients; antagonism of other bacteria through the production of substances which inhibit or kill non-indigenous species; stimulation of development of certain tissues in the GI tract; stimulation of production of natural antibodies. ${ }^{3}$

\section{Harmful effects of the normal flora}

Bacterial synergism between a member of the normal flora and a potential pathogen; competition for nutrients; induction of a low-grade toxemia; the normal flora may be agents of disease and transfer to susceptible hosts. ${ }^{6,7}$

\section{Ideal properties}

The ideal synbiotic should possess these characters together with the following features which the present knowledge of this subject suggests would be desirable: must belong to the natural flora; non-pathogenic and nontoxic; should produce antibacterial substances; beneficial to the host animal in some way; of high viability; suppress growth of toxic bacteria; stable on storage and in the field; able to survive in or colonize the gut; amenable to cultivation on an industrial scale; and have palatable taste and flavor. , $^{6,7}$

The FAO and the WHO have recommended that probiotic bacterial strains be characterized by their spectrum of resistance to antibiotics, their metabolic and hemolytic activities, their capacity to produce toxins, their infectious power in immunosuppressed animal models and their side effects in humans.

\section{Probiotics and immune system modulation}

The cellular interactions are enhanced by adhesion molecules, and the activated cells release different cytokines. These complex cellular interactions induce a systemic immune response. If the antigen penetrates by the oral route, a secretory immune response is obtained, which is mediated by secretory IgA. The effects of lactic acid bacteria on the systemic immune response and on the secretory immune system have been researched widely. Potential health benefits of lactic acid bacteria include protection against enteric infections, use as an oral adjuvant, the immunopotentiator in malnutrition, and the prevention of chemically induced tumors.

Probiotics modulate host cell signaling pathways, including Akt, mitogen-activated protein kinases, and nuclear factor-kappa $B$ to mediate these intestinal epithelial functions. The major immunological endpoints that have been evaluated include: host defense as it relates to resistance against infection; B-lymphocyte activity including $\operatorname{IgM}$ and IgA production; phagocytes as it relates to phagocytic activity among other parameters; $\mathrm{T}$ lymphocytes as measured by natural killer (NK) cell activity; Hematopoiesis as reflected by monocyte to macrophage ratios and macrophage cell levels; and finally allergic reaction measured by IgE production and Th1 or Th2 cell-associated cytokine production.

Some probiotic microorganisms are also able to increase the IgA cycle, and this effect is dose dependent. Perdigon and Alvarez have reviewed the effects of probiotics on the immune state. Isolauri et al, have reviewed the interaction between probiotics and the gastrointestinal immune system. T-independent IgA induction was also demonstrated; the cytokines transforming growth factor (TG), interleukin-4 (IL-4) (50), and IL-2, IL-6, and IL-10 work in a synergistic way from other immune cells different from $\mathrm{T}$ cells and can promote the switch from $\operatorname{IgM}$ to $\operatorname{Ig} \mathrm{A}$ expression. The stimulation with probiotic 
bacteria induced signals on epithelial and immune cells that evoked different patterns of cytokines in the intestine depending on the dose administered. Some of the major findings are reviewed below. Subcutaneous injections of heat killed L. casei strain shirota to BALB/C, C3H/He, $\mathrm{C} 57 \mathrm{BL} / 6$, athymic nu/nu or nu/+ enhanced resistance to systemic C. monocytogenes infection. Swiss albino mice fed live L. casei were protected against Salmonella typhimuriun infection and secretory $\operatorname{IgA}$ in intestinal fluid was elevated. ${ }^{8}$

\section{Production of Antimicrobial Substance}

Most bacterial probiotics produce organic acids, such as acetic and lactic acid, which can act as bacterial growth inhibitors. Some probiotics, for example, lactobacillus, produce hydrogen peroxide when transferred from an anaerobic to aerobic environment. Hydrogen peroxide can inhibit the growth of pathogenic microorganisms. In addition to these general antimicrobials produced by probiotics, there is a long list of other products that have been shown to have antimicrobial activity. L. acidophilus has been shown to produce substances between the molecular weights of 200 and 6,200, some of which are sensitive to proteases and can inhibit staphylococcus, streptococcus, E. coli, and salmonella. Lactobacillus rhamnosum strain GG(LGG) produces a broad spectrum low molecular weight centimicrobial peptide which is plasmid mediated and has activity against staphylococcus, streptococcus, mycobacterium, bacillus, clostridium, listeria, bifidobacterium, E. coli, and salmonella. There have been numerous other probiotics that have been shown to produce antimicrobials including: L. bulgarius, L. reuteri, L. helveticus, L. fermentation, L. plantarum, Lactococcus lactis, and S. Cremoris. ${ }^{9}$

\section{Oral benefits}

Probiotic organisms have several decided general health benefits, which includes: assists such other natural antimicrobial agents as echinacea, goldenseal, and garlic during intestinal cleansing programs. Assists liver in the detoxification process, thereby helping to prevent such liver diseases as hepatic encephalopathy (severe liver dysfunction). Bolsters the immune system. Decreases incidence and duration of diarrhea, whether it is caused by antibiotics, Clostridium difficile, rotaviral, or other pathogens. Enables better growth development in children. Have a positive influence on autistic children. Have anticarcinogenic, anti-mutagenic, and anti-allergic activities. Helps to alleviate such inflammatory conditions as Crohn's disease and ulcerative colitis. Helps to prevent osteoporosis. Helps to prevent colon cancer by preventing the breakdown of enzymes that contribute to the growth of cancer-causing agents. Helps to alleviate food, chemical, and/or environmental sensitivities. Improves digestion and balance cholesterol metabolism. Increase nutritional value of foods through better digestibility and an increased absorption of nutrients. Influence better intestinal and urogenital flora, especially after antibiotic and radiation therapies, which are known to induce colitis, yeast infections, and vaginitis. Maintains mucosal integrity. Manufactures, process, and helps to absorb nutrients, especially the B-vitamins. Manufactures lactase, which promotes intestinal lactose digestion. Prevents and reduces intestinal tract infections, including those caused by bacteria or viruses, candida, and Helicobacter pylori. Promotes a feeling of well-being. Provides an antagonistic environment for pathogens by normalizing beneficial organisms. This encourages friendly ones to crowd out of harmful ones, thereby blocking their adhesion sites in addition to inactivating enterotoxins. Reduces catabolic products (bile pigments) eliminated by kidney and liver. Reduces and eliminates overgrowth of small bowel bacteria. Regulates gut motility, thereby reducing such conditions as constipation. Alleviate side-effects caused by antibiotic therapy. ${ }^{10}$

\section{CONCLUSION}

Synbiotics represent a new area of research in oral medicine, the examination of the close relationship 158/ips between food and oral health. Preliminary data obtained by various research laboratories have been encouraging, but numerous randomized clinical studies will be required to clearly establish the potential of synbiotics in preventing and treating oral infections. There is a great need to elucidate the role of the oral beneficial microbiota, to identify beneficial bacteria and to conduct proper large-scale studies on the usefulness of synbiotics to maintain or improve oral health.

Funding: No funding sources Conflict of interest: None declared Ethical approval: Not required

\section{REFERENCES}

1. Gill HS, Rutherfurd KJ, Prasad J, Gopal PK. Enhancement of natural and acquired immunity by Lactobacillus rhamnosus, Lactobacillus acidophilus and Bifidobacterium lactis. British $\mathrm{J}$ Nutr. 2000;83(2):167-76.

2. Teughels W, Van EM, Sliepen I, Quirynen M. Probiotics and oral healthcare. Periodontol 2000. 2008;48:111-47.

3. Stark CA, Edlund C, Sjöstedt S, Kristensen G, Nord CE. Antimicrobial resistance in human oral and intestinal anaerobic microfloras. Antimicrob Agents Chemother. 1993;37(8):1665-9.

4. Gibson GR, Ottaway PB, Rastall RA. Prebiotics: new developments in functional foods. Oxford: Chandos Publishing ltd; 2000.

5. Floch MH. Use of diet and probiotic therapy in the irritable bowel syndrome: analysis of the literature. J Clin Gastroenterol. 2005;39(5):243-6.

6. Berg RD. Probiotics, prebiotics or 'conbiotics'? Trends Microbiol. 1998;6(3):89-92.

7. Food and Health Agricultural Organization of the United Nations and World Health Organization. 
Guidelines for the evaluation of probiotics in food, 2002. Available at: ftp://ftp.fao.org/es/esn/food/wgreport2.pdf. Accessed on 12 February 2021.

8. Ahola AJ, Yli-Knuuttila H, Suomalainen T, Poussa T, Ahlström A, Meurman JH, et al. Short-term consumption of probiotic-containing cheese and its effect on dental caries risk factors. Arch Oral Biol. 2002;47(11):799-804.

9. Montalto M, Vastola M, Marigo L, Covino M, Graziosetto R, Curigliano V, et al. Probiotic treatment increases salivary counts of lactobacilli: a double-blind, randomized, controlled study. Digestion. 2004;69(1):53-6.
10. Nikawa H, Makihira S, Fukushima $H$, Nishimura $H$, Ozaki Y, Ishida K, et al. Lactobacillus reuteri in bovine milk fermented decreases the oral carriage of mutans streptococci. Int $\mathrm{J}$ Food Microbiol. 2004;95(2):219-23.

11. Socransky SS, Haffajee AD. Periodontal microbial ecology. Periodontol. 2005;38:135-87.

Cite this article as: Mehta K, Mehta H. Synbiotics in oral immunity: a review. Int J Otorhinolaryngol Head Neck Surg 2021;7:915-8. 